D. Revista Humanismo

NO y Sociedad

\title{
Río Grande de La Magdalena: un sueño llamado hidrovía.
}

Rio Grande de La Magdalena: a dream called waterway.

Leslio Ricardo González ${ }^{1 \star}$, Adm. Negocios Internacionales.

${ }^{1}$ Directivo de la Fundación Colombiana de Suboficiales (FUNCOLSUB) Cartagena; Suboficial Jefe Técnico en uso de buen retiro de la Armada Nacional de Colombia; Diplomado en transporte marítimo, fluvial y legislación portuaria, Sociedad de Ingenieros del Atlántico y Pronaval; Diplomado Visión Competitiva, Desafío Empresarial y Solidario, Universidad Fitec y Funcolsub.

Aceptado: noviembre 30 de 2012.

\section{Resumen.}

El propósito de la navegación fluvial, consiste en prestar servicios de transporte en embarcaciones adecuadas en cuanto a tipo y tamaño, la eficiencia de estos servicios depende de los medios de producción que se utilicen, del uso que de ellos se haga, de las infraestructuras de las vías de navegación y sus puertos interiores. Las potencialidades que ofrece Colombia en cuanto a riquezas hídricas, la ubicación de la cuenca del Río Grande de La Magdalena, su óptimo aprovechamiento como modo de transporte fluvial, podría darse al ejecutarse el proyecto hidroviario que contempla el plan de desarrollo del gobierno actual, permitiendo el desplazamiento de un mayor volumen de carga y pasajeros, economías en combustible, alivio al modo carretero, descontaminación, entre otros, posicionando al país en un significativo ascenso en el ranking competitivo latinoamericano, en el presente informe se describen las características de una hidrovía y los parámetros que debe tener el Río Grande de la Magdalena para ser una hidrovía.

Palabras clave: competitividad, hidrovía, optimización.

\section{Abstract.}

The purpose of river navigation is to provide transport services in suitable vessels according to their type and size. The efficiency of these services depends on the means of production, its use, and the infrastructure of waterways and inland ports. Colombia offers an interesting water potential considering the location of the watershed of the Rio Grande de La Magdalena and the use of this river for transport. This potential could come to reality with the waterway project incuded in the development plan of the current government. This project would allow moving a greater volume of freight and passengers with fuel economies, relief of road traffic, and decontamination, among others. The project would help

Para citar este artículo: Ricardo L. Río Grande de La Magdalena: un sueño llamado hidrovía. Rev Humanismo y Sociedad, 2013; Volumen 1: 81-85.

${ }^{*}$ Autor para correspondencia: Leslio Ricardo González. Fundación Colombiana de Suboficiales (FUNCOLSUB), Cartagena

E-mail: lesrigon@yahoo.com

Revista. Humanismo.Soc. 2013; 25: 81-85. 
colombian competitiveness to rank better in the Latin American context. This paper describes the characteristics of such waterway and the required parameters of the Río Grande de la Magdalena to meet this objective.

Key words: competitiveness, resource optimization, waterway.

\section{Introducción.}

Desde lo histórico, el Río Magdalena ha sido protagonista en el desarrollo económico, social, cultural, local, regional, nacional; hoy en día está enmarcado en una grave problemática que pasa desde el uso inadecuado de recursos naturales, inundaciones, sequias, contaminación, pobreza de las poblaciones rivereñas, tensiones sociales, deterioro ambiental, conflictos de uso del suelo, asentamientos en áreas de riesgo, entre otros (Zambrano, 1979; Bernal, 1996).

Con el transcurrir del tiempo, en distintos periodos de gobierno, el Río Magdalena ha sido objeto de permanentes evaluaciones y proyectos, intentado encontrar esquemas que permitan llevarlo nuevamente a ese rol protagonista, sin resultado alguno (Bernal, 1996; Kaufmann, 1973).

La necesidad de aumentar la seguridad y disminuir el costo de los fletes en el transporte de carga por carretera, ha impulsado algunos empresarios a explorar el

Potencial del Río Grande de la Magdalena. Empresas principalmente Sofasa y otras como Familia, Noel, Corona, Argos e Incolmotos decidieron experimentar, los empresarios se dan cuenta de que pueden reducir sus fletes utilizando el modo de transporte fluvial como alternativa complementaria al modo carretero (Alfredo, 2008).

El Plan Nacional de Desarrollo (PND) 2010-2014, contempla la recuperación del rio Magdalena, articulado a los corredores de la prosperidad y convertirlo así en el principal proyecto hidroviario de Colombia; pero ¿será posible para los colombianos cristalizarse el sueño de contar con Hidrovía?

Es necesario verificar si existen autopistas, que son una vía de circulación de automóviles y vehículos terrestres de carga; es rápida y segura y admite un volumen de tráfico considerable, y se diferencia de una carretera porque la autopista es de una sola dirección (Wikipedia, 2013).
Las primeras vías construidas con esta configuración se hicieron en Italia durante los años veinte del siglo XX. Para poder ser calificada como autopista, una vía de circulación debe reunir las siguientes características (Wikipedia, 2013).

1. Dos bandas de circulación, una para cada sentido, separadas entre sí por una franja ancha de terreno o por vallas de protección.

2. Al menos dos carriles de circulación en cada banda.

3. Arcenes laterales en cada banda, para que un vehículo pueda detenerse en caso de emergencia sin obstaculizar el tráfico.

4. Curvas poco pronunciadas para que los vehículos no tengan que aminorar la marcha al circular por ellas.

5. Ausencia de cruces a nivel, que se resuelven mediante pasos superiores o inferiores.

6. Entradas y salidas con carriles, separados de los principales, de desaceleración y de aceleración para que los vehículos que salen o entran en la autopista cambien su velocidad fuera de ella.

7. Dos bandas de circulación, una para cada sentido, separadas entre sí por una franja ancha de terreno o por vallas de protección.

8. Las salidas y entradas están situadas casi siempre en el lado derecho en el sentido de la marcha, ya que el carril izquierdo es el de adelantamiento y, por lo tanto, el más rápido.

Así las cosas, se pregunta ¿existen en Colombia autopistas? ¿Qué se requiere para tener una Hidrovía? es totalmente cierto que luego del marítimo, el transporte fluvial es el modo que mayor economía a escala produce. No obstante esas ventajas se 
pierden cuando la vía navegable interior no reúne las condiciones necesarias para su total aprovechamiento, de tal manera, el modo y las condiciones de la ruta, en nuestro caso los ríos, deben desarrollarse en forma armónica como un todo, siendo así la Hidrovía, una vía fluvial navegable por barcos de tamaño adecuado, teniendo en cuenta la profundidad, el ancho, el lecho de la corriente y la morfología del terreno (Monserrat, 2005).

La hidrovía es un camino dentro de un sistema de aguas interiores o continentales que posibilita el desplazamiento de diversas embarcaciones, actuando como una vía de transporte fluvial que permite una importante interconexión comercial (Pronaval, 2008)

En estos tiempos en donde el transporte fluvial se incrementa a pasos agigantado, contar con una vía hídrica debe ser objeto del máximo provecho posible; es por ello que semejante regalo de la naturaleza debe ser utilizado con el mejor criterio e inteligencia posible para el desarrollo de la misma.

En este sentido, un proyecto hidroviario, tiene como meta realizar obras de profundización, rectificación, ensanche y señalización de las vías fluviales de navegación, asegurando una normal navegación, permitiendo el uso permanente de la Hidrovía los 365 días del año, durante las 24 horas del día (Pronaval, 2008).

Cáceres (2008), afirma que algunos de los objetivos que debe cumplir una hidrovia son los siguientes:

1. Optimizar las condiciones de navegabilidad, ofreciendo un corredor económico y seguro para la exportación e importación.

\section{Servir de integración regional.}

3. Permitir la interiorización de los cursos fluviales y el crecimiento de transporte masivo de grandes volúmenes, realizados a bajos costo

4. Preservar las condiciones de seguridad, para que los cambios ecológicos no generen contaminación, arrastres sedimentarios o crecidas depredatorias continuas, originadas por las alteraciones de régimen natural de las vías fluviales.
Pronaval (2008), describe que para poder ser calificado como Hidrovía, una rio de circulación debe reunir las siguientes características:

1. Canal estandarizado: profundidad - ancho - radios curvatura.

2. Servicio de inspección y cartografía.

3. Señalización diurna - nocturna.

4. Comunicaciones y control de tráfico.

5. Infraestructura asistencial.

Capra K. (2003) destaca la utilización de las hidrovías y asevera que repercute en beneficios como: bajo consumo de combustible, gran capacidad de manejo de carga, manejo de grandes distancias, ambientalmente amigable, alto grado de seguridad, económico en términos de energía no renovable, ayuda a aliviar la red de carreteras (Figura 1).

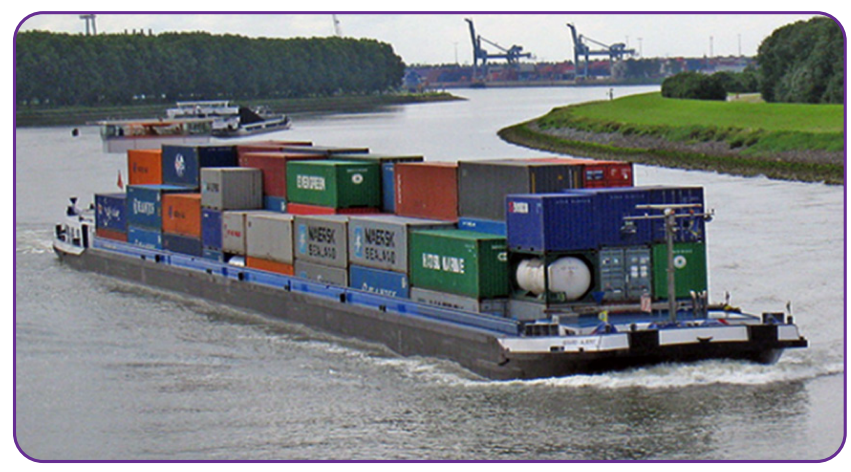

Figura 1. Embarcaciones modernas con impulsador incorporado adecuado para navegar por hidrovías. Fuente: Nuestro Mar 2010.

En el libro Voiles en seine (1989), se muestra el puerto fluvial en la ciudad de Rouen, sobre la vertiente del Sena en Francia integrada a la red hidroviaria de Europa, en la celebración de los doscientos años de la independencia de Francia, realizó uno de los primordiales eventos de la celebración, con un encuentro de los principales veleros del mundo, denominado Les voiles de la liberte, sin descuidar las operaciones normales del puerto, cubrió con gran éxito la logística del evento, demostrando la eficiencia y eficacia con que operan las hidrovías (Figura 2). 


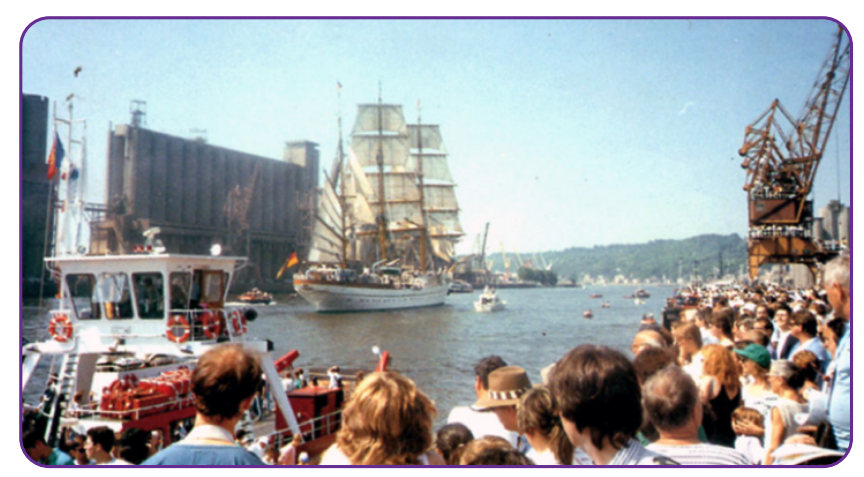

Figura 2. Evento Les Voiles de la liberte donde se muestra la eficacia con que operan las hidrovías. Fuente: Voiles en seine, 1989.

Se puede apreciar en las figuras la capacidad de instalaciones portuarias que posee el puerto, además el buque escuela Gloria de la Armada de Colombia como invitado (Figuras 3 y 4 ).

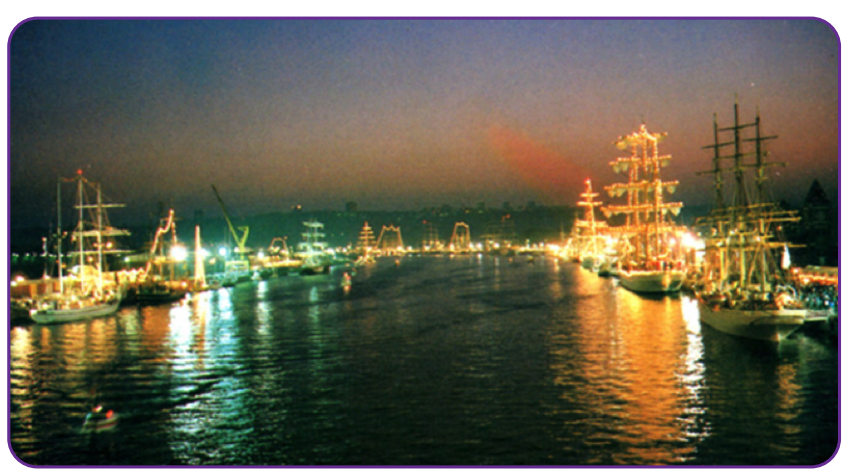

Figura 3. Capacidad de instalaciones de servicios portuarios. Fuente: Voiles en seine

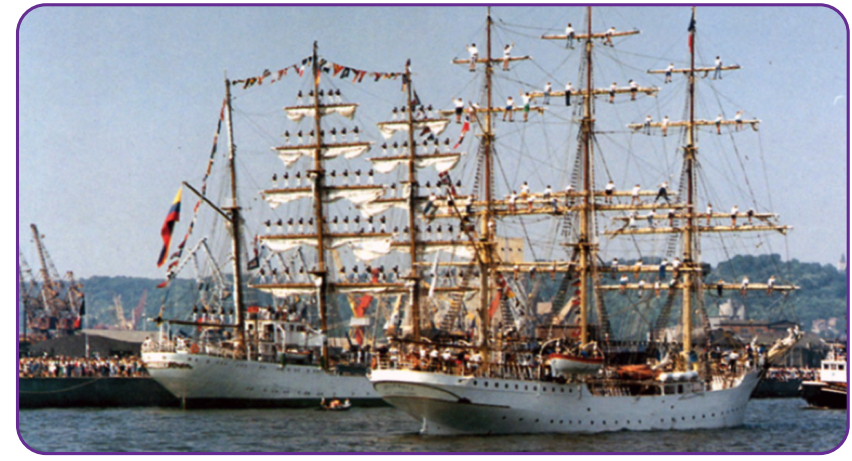

Figura 4. Buque escuela Gloria de la Armada de Colombia. Fuente: Voiles en seine, 1989.

Tratando de asimilar en Colombia, es como si la ciudad de Barrancabermeja uno de los principales puerto sobre el rio Magdalena, hubiese realizado el evento Sail Cartagena, encuentro de veleros en homenaje al bicentenario de la independencia del país.

Siguiendo los parámetros citados se pregunta ¿seguirá el sueño de Hidrovía para los colombianos?

\section{Conclusión.}

Unodelos principales obstáculos queenfrenta Colombia para mejorar su competitividad es precisamente las falencias en materia de infraestructura, particularmente en logística de transporte y en este aspecto desarrollar el proyecto hidroviario cuenca del Río Grande de la Magdalena es la variable más importante a la solución.

\section{Referencias}

Bernal G. Caracterización morfológica de la llanura deltáica del Río Magdalena con énfasis en el sistema lagunar de la ciénaga grande de Santa Marta Colombia Mitt. Inst. ColomboAlemán Invest. Cient, 1996;25:19-48.

Cantello, Alfredo. Noviembre 21/2008 Puertos y Vías Fluviales del Río Magdalena http://www.skyscrapercity.com/ showthread.php? $p=31715892$.

Cáceres Duarte S. Logística sobre el agua. Revista de logística. 2010 May;9:1-4.

Capra K. La Hidrovía Paraná Paraguay Una alternativa a los puertos del Pacifico.http://www.udape.gob.bo/portales_html/
Documentos\%20de\%20trabajo/DocTrabajo/2003/HPP1.pdf

Departamento Nacional de Planeación. http://www.dnp.gov. $\mathrm{col}$

Kaufmann $R$, Hevert F. El régimen fluviométrico del Río Magdalena y su importancia para la Ciénaga Grande de Santa Marta. Mitt. Inst. Colombo-Alemán Invest. Cient, 1973;7:121137.

Monserrat Llairo M. La Hidrovía Paraguay-Paraná: realidades y controversias. Facetas de la relación política - territorio. 2005; 1(6).

Pronaval. Sociedad de ingenieros del Atlántico. Transporte

Revista. Humanismo.Soc. 2013; 25: 81-85. 
Marítima Fluvial y Legislación Portuaria, documento módulos hidrovias, Vertientes hidrográficas.

2008. Capítulo 5.

Pronaval. Sociedad de ingenieros del Atlántico. Transporte Marítima, Fluvial y Legislación Portuaria, documento módulos hidrovias, Vertientes hidrográficas. 2008 capitulo 7.
Voiles en seine, edition du ptit normand 1989.p. 100-103.

Wikipedia. http://es.wikipedia.org/wiki/Autopista definición e historia. Feb 2013.

Zambrano F. La navegación a vapor por el rio Magdalena. Anuario Colombiano de Historia Social y de la Cultura. 1979; 9:63-75. 